\title{
Velocity Control of Unmanned Helicopter Based on Fuzzy-PID
}

\author{
Chen Xianxiang ${ }^{1}$,Liu Ying ${ }^{1}$, Hu Xiongwen ${ }^{1}$,Qian \\ $\mathrm{Yi}^{1}$ \\ Beijing Institute of Computer Application \\ Beijing,China \\ chenxx_710@126.com
}

\author{
Wang Jinhua ${ }^{2}$ \\ China academy of ordnance science \\ Beijing,China
}

\begin{abstract}
The fuzzy-PID control approach was used on the velocity control of the unmanned helicopter. Furthermore, a novel parameter self-tuning method was presented that the parameters of the PID controller were self-tuned based on the parameters of the last control period according to the deviation and its variation ratio, adequately considering the control information of the last control period. The increments of the PID parameters were calculated by the fuzzy inference system. Matlab simulation and the hardware-in-loop simulation were performed, the simulation results verified the feasibility and the effectiveness.
\end{abstract}

Keywords-fuzzy-PID; self-tuning; unmanned helicopter; hardware-in-loop simulation

\section{INTRODUCTION}

The conventional PID controller was easy to use, however, it can't simultaneously meet the static and dynamic performance, it always had to balance the tracking ability and the disturbance restraining ability, so the control system can't achieve the optimal control effect. The fuzzy PID control combined the merits of the conventional PID, like that easy to use and didn't rely on the controlled plant, and the strong robustness of the fuzzy control, so the fuzzy PID was chosen for the velocity control of the unmanned helicopter. The fuzzy self-tuning PID control tuned the PID parameters online according to the deviation and its variation ratio based on the fuzzy rules, meeting the requirement that the PID parameters should meet the deviation and its variation ratio at different time.

The Reference[1] designed the attitude controller of the helicopter integrating the conventional PID and the fuzzy PID, the fuzzy PID was used to suppress the overshoot. Reference[2],[3] designed the attitude controller of the helicopter utilizing the fuzzy PID control approach, the controller parameters were tuned by the fuzzy inference directly, it was a position-type tuning method. For such position-type self-tuning method, a certain deviation and its variation ratio correspondingly decided a certain group of parameters of PID controller. Actually, the deviation and its variation ratio shown the control effect of the last control period. The position-type self-tuning method ignored the control information of the last control period, so the parameters obtained was not always suitable for current control, therefore, the increment self-tuning approach was presented in this paper.

\section{SELF-TUNING FUZZY PID CONTROLLER DESIGN}

A. Self-tuning Fuzzy PID Control Structure

The discrete PID control algorithm was written as

$$
\begin{aligned}
u(k)= & K_{p} e(k)+K_{i} T \sum_{j=0}^{k} e(j) \\
& +K_{d} \frac{e(k)-e(k-1)}{T}
\end{aligned}
$$

Where, $k$ was the sample sequence number, $T$ was the sample time period.

It can be seen from the above formula that the three gain parameters played an important role in the PID control algorithm. The three gain parameters were self-tuned by the fuzzy inference system according to the fuzzy PID control structure shown in Fig.1, where, $K_{e}, K_{e c}$ were quantization factors, $k_{k p}, k_{k i}, k_{k d}$ were scaling factors.

The increment self-tuning approach was presented for the three gain parameters $K_{p}, K_{i}, K_{d}$, namely, the current values of the three parameters were calculated according to the PID parameters of the last control period, so it was to meet the control requirement and improve the control effect, shown in equation (2)-(4).

$$
\begin{aligned}
& K_{p}(k)=K_{p}(k-1)+\Delta K_{p}(k) \\
& K_{i}(k)=K_{i}(k-1)+\Delta K_{i}(k) \\
& K_{d}(k)=K_{d}(k-1)+\Delta K_{d}(k)
\end{aligned}
$$

Where, $k$ was the sample sequence number, $\Delta K_{p}(k) \Delta K_{i}(k) \Delta K_{d}(k)$ which obtained by the fuzzy inference system were increments for calculating the current values of the PID parameters.

\section{B. Fuzzy Inference System Design}

The Mamdani-type inference was used for the fuzzy inference system, and the universe of the discourse of the input and output variables were defined as

$$
e, e c, \Delta K_{p}, \Delta K_{i}, \Delta K_{d}=\{-3,-2,-1,0,1,2,3\}
$$

Accordingly, the fuzzy linguistic variable sets were presented in equation (6). 


$$
e, e c, \Delta K_{p}, \Delta K_{i}, \Delta K_{d}=\{N B, N S, Z, P S, P B\}_{(6)}
$$

The linguistic variable levels were assigned as $N B, N S, Z, P S, P B$ in the subsets represented as negative large, negative small, zero, positive small, positive large, respectively.

The fuzzy rules were built according to the properties of the PID controller and the relationship of the control effect and the deviation and its variation ratio, the basic principle of building fuzzy rule was that if the sign of the deviation and its variation ratio were the same, which indicated that the current speed was apart from the desired speed, then it should augment the $K_{p}$ and $K_{d}$; if the sign was different which indicated approaching to target, then it should decrease or hold the $K_{p}$ and $K_{d}$ according to the value of the deviation and its variation ratio; The parameter $K_{i}$ was mostly relate to the deviation, a biggish deviation indicated that it should be decrease and a small deviation indicating augment. Therefore, the parameters self-tuning fuzzy rules were built shown in table 1 . The rules of $\Delta K_{p}, \Delta K_{i}, \Delta K_{d}$ were in turns in each cell.

Since the Triangular-shape membership function was facility for use, it was chosen in this paper. The graph of the membership functions were shown in Fig.2 to Fig.3.

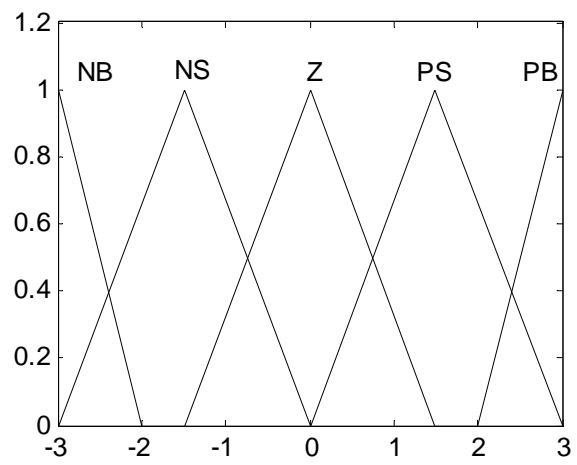

Figure 2. Membership function of e and ec

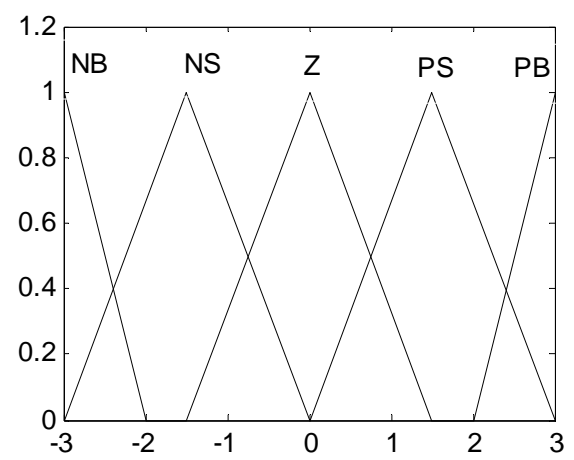

Figure 3. Membership function of the PID parameters
The universe of physical sets regarded as velocity deviation $[-3 \mathrm{~m} / \mathrm{s}, 3 \mathrm{~m} / \mathrm{s}]$ and velocity deviation ratio $[-3 \mathrm{~m} / \mathrm{s} 2$, $3 \mathrm{~m} / \mathrm{s} 2]$, therefore defined the quantization factor as

$$
k_{e}=n_{e} / e, k_{e c}=n_{e c} / e c
$$

where,

$$
\begin{aligned}
& n_{e}= \begin{cases}-3(1+\alpha), & k_{e} e<-3 \\
(1+\alpha) e, & \left|k_{e} e\right|<3 \\
3(1+\alpha), & k_{e} e>3\end{cases} \\
& n_{e c}= \begin{cases}-3(1+\beta), & k_{e c} e c<-3 \\
(1+\beta) e c, & \left|k_{e c} e c\right|<3 \\
3(1+\beta), & k_{e c} e c>3\end{cases}
\end{aligned}
$$

The quantization factor ensured that the regarded universe of physical sets matched the universe of fuzzy sets.

The quantization factor and the scaling factor not only provided mappings from the universe of physical sets to the universe of fuzzy sets, but also regulated the control performance of the control system. The $K_{e}$ and $K_{e c}$ were related to $\alpha$ and $\beta$ respectively, so it was convenience that changing the values of $\alpha$ and $\beta$ to change the values of $K_{e}$ and $K_{e c}$.And if $\alpha=0, \beta=0$, then the universe of physical sets exactly match the universe of fuzzy sets. The alternation of the $K_{e}$ altered the deviation control effect, therefore, different $K_{e}$ may obtain different control performance of the system, like rise rate, overshoot, and static deviation. The alternation of the $K_{e}$ altered the deviation ratio control effect, therefore, different $K_{e c}$ may obtain different ability of restraining the variance of the states. The scaling factor regulated the variance rate of the PID parameters, namely it was the sensitivity of the PID parameters to the deviation and its variation ratio. After numerous simulation experiments, finally, the quantization factors and the scaling factors in each channel were chosen

as, forward speed channel : $k_{k p}=0.01, k_{k i}=0.05$ $k_{k d}=0.01, \alpha=0, \beta=0$; lateral speed channel: $k_{k p}=0.005, k_{k i}=0.1, k_{k d}=0.01, \alpha=0, \beta=0$.

\section{Simulink Simulation}

Utilize the Matlab FuzzyLogicToolBox[5] to simulate the unmanned helicopter speed fuzzy PID control system. The range of each PID parameter was determined based on the simulation on PID controller to obtain a feasible rule bases with high inference efficiency. The tunable range of the PID parameters $K_{p}, K_{i}, K_{d}$ in forward speed 
channel was set as [0.01, 0.3], [0, 0.1], [0, 0.15] respectively, PID parameters $K_{p}, K_{i}, K_{d}$ in lateral speed channel was set as $[0.01,0.3],[0,0.1],[0,0.1]$, respectively. The step responses shown in the Fig.4, where, the dashed-dot line PID1 and the dashed line PID2 were the step responses of the conventional PID controller. The parameters of the PID1 were set as, forward speed channel: $K_{p=0.12,} K_{i=0.03 \text {, }}$ $K_{d=0.01 \text {, lateral speed channel: }} K_{p=0.12,} K_{i=0.03}$, $K_{d}=0.01$. The parameters of the PID2 were set as, forward speed channel: $K_{p=0.08}, K_{i=0.01}, K_{d=0.01 \text {; Lateral }}$ speed channel: $K_{p=0.08,} K_{i=0.02}, K_{d=0.006 \text {; }}$ The solid line FPID was the step response of the fuzzy PID controller. It was shown in Fig.4 that the control system with the fuzzy controller obtained fast response and zero overshoot.
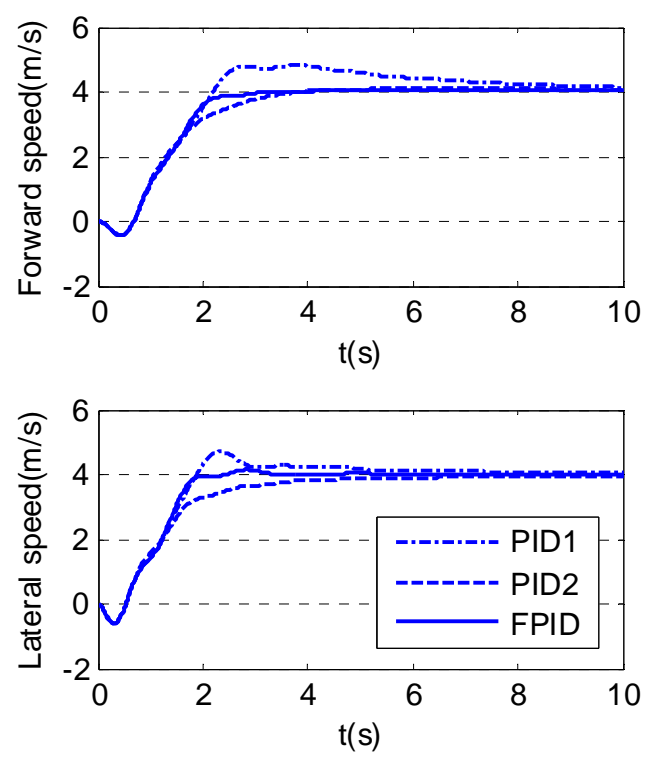

Figure 4. Step response of the forward and lateral speed

\section{UNMANNED HELICOPTER HARDWARE- IN-LOOP SIMULATION VERIFICATION}

The unmanned helicopter hardware-in-loop simulation structure was depicted as Fig.5. The simulation system was consisted of ground control station, onboard computer, helicopter, and 3 dimension(3D) visual simulation system. The ground control station provided the command speed, the onboard computer contained fuzzy PID controller and the inner loop $H_{\infty}$ loop-shaping robust controller, the 3D visual simulation system simulated the dynamics of the unmanned helicopter, and feedback the flight states. The 3d visual simulation system with high fidelity took the environment factors into consideration, it was in favor of the real flight. The action of the rudders could be observed by the unmanned helicopter practicality.

The Matlab code generation block was used to obtain the C code of the PID control algorithm, which was explanted to the onboard computer, and the hardware-in-loop simulation was executed. The flight trajectory was shown in Fig.6, where the 3 dimension helicopter model was shown on the top right corner.

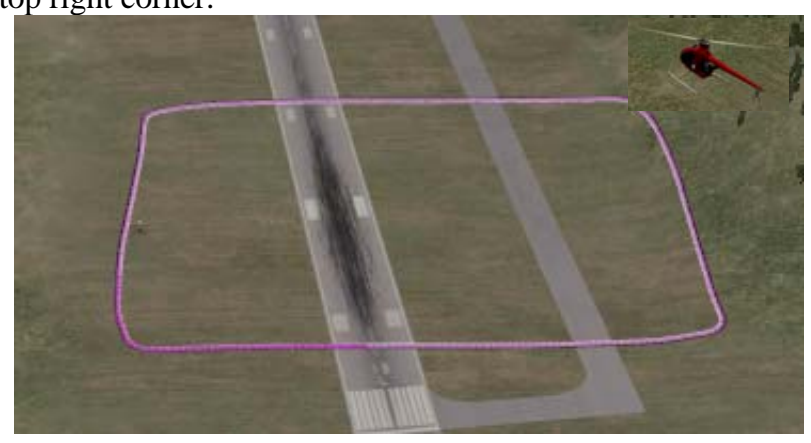

Figure 6. Flight trajectory

\section{CONCLUSION}

The fuzzy PID control involved the advantages of the conventional PID control that it was easy for utilization and it didn't rely on the precise mathematical model of the controlled plant, and the strong robustness of the fuzzy control. The fuzzy PID control was propitious to the outerloop control, it was in favor of improving the robustness of the unmanned helicopter. The increment self-tuning approach was presented, the current PID parameters were self-tuned online according to the deviation and its variation ratio based on the PID parameters of the last control period. The simulation results shown the feasibility and effectiveness.

\section{REFERENCES}

[1] Le Zhang, Shaojie Bi, Hong Yang. Fuzzy-PID Control Algorithm of the Helicopter Model Flight Attitude Control, Control and Decision Conference (CCDC), 2010, Chinese, 1438 - 1443.

[2] Dai Meihong. Attitude control of helicopter model based on fuzzy PID control[D]. Northeastern university, 2005.

[3] Ji Jian, Jiang Ju. A model helicopter self-retun system based on fuzzy PID control[J]. Aircraft design, 2011, 31(1): 61-66.

[4] Zhang Defeng. MATLAB fuzzy system design, national defense industry press, 2009.

[5] Fuzzy Logic Toolbox User’s Guide, Mathworks, 2010. 


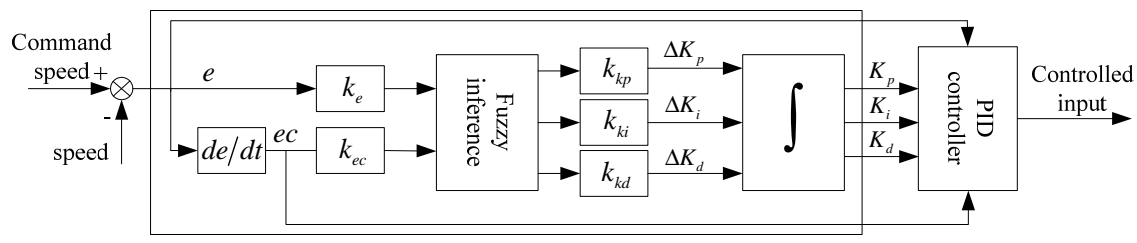

Figure 1. Self-tuning fuzzy PID controller structure

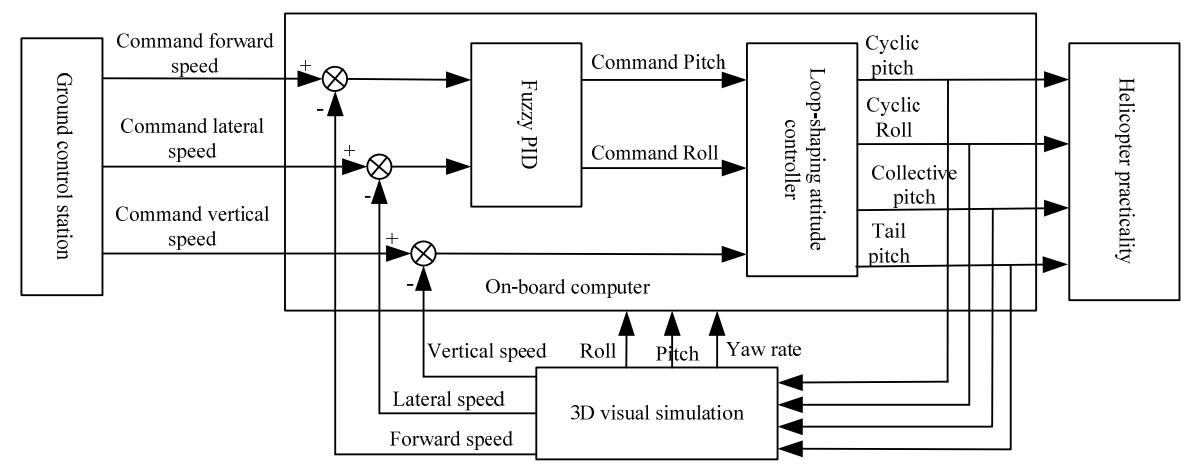

Figure 5. Hardware-in-loop simulation system

TABLE I RULES OF $\Delta K_{p}, \Delta K_{i}, \Delta K_{d}$

\begin{tabular}{|c|c|c|c|c|c|}
\hline$e C$ & $\mathbf{N B}$ & $\mathbf{N S}$ & $\mathbf{Z}$ & $\mathbf{P S}$ & PB \\
\hline $\mathrm{NB}$ & & & & & \\
\hline $\mathrm{NS}$ & $\mathrm{PB}|\mathrm{NB}| \mathrm{PB}$ & $\mathrm{PB}|\mathrm{PS}| \mathrm{PB}$ & $\mathrm{NB}|\mathrm{PB}| \mathrm{PB}$ & $\mathrm{NS}|\mathrm{PS}| \mathrm{NB}$ & $\mathrm{Z}|\mathrm{NB}| \mathrm{NB}$ \\
\hline $\mathrm{Z}$ & $\mathrm{PB}|\mathrm{NB}| \mathrm{PS}$ & $\mathrm{PS}|\mathrm{PS}| \mathrm{PS}$ & $\mathrm{NS}|\mathrm{PS}| \mathrm{PS}$ & $\mathrm{NS}|\mathrm{PS}| \mathrm{NS}$ & $\mathrm{PS}|\mathrm{NB}| \mathrm{NS}$ \\
\hline $\mathrm{PS}$ & $\mathrm{PS}|\mathrm{NB}| \mathrm{NS}$ & $\mathrm{PS}|\mathrm{PS}| \mathrm{NS}$ & $\mathrm{Z}|\mathrm{PS}| \mathrm{PS}$ & $\mathrm{PS}|\mathrm{PS}| \mathrm{NS}$ & $\mathrm{PB}|\mathrm{NB}| \mathrm{NS}$ \\
\hline $\mathrm{PB}$ & $\mathrm{Z}|\mathrm{NB}| \mathrm{NB}$ & $\mathrm{NB}|\mathrm{PS}| \mathrm{NB}$ & $\mathrm{NS}|\mathrm{PS}| \mathrm{PS}$ & $\mathrm{PS}|\mathrm{PS}| \mathrm{PS}$ & $\mathrm{PB}|\mathrm{NB}| \mathrm{PS}$ \\
\hline
\end{tabular}

